Voix et Images

voixetimages

\title{
"La poésie dans la prose, ou le clochard illuminé »
}

\section{André Brochu}

Volume 12, numéro 2 (35), hiver 1987

Jacques Brault

URI : https://id.erudit.org/iderudit/200627ar

DOI : https://doi.org/10.7202/200627ar

Aller au sommaire du numéro

\section{Éditeur(s)}

Université du Québec à Montréal

\section{ISSN}

0318-9201 (imprimé)

1705-933X (numérique)

Découvrir la revue

\section{Citer cet article}

Brochu, A. (1987). « La poésie dans la prose, ou le clochard illuminé ». Voix et Images, 12(2), 212-220. https://doi.org/10.7202/200627ar d'utilisation que vous pouvez consulter en ligne.

https://apropos.erudit.org/fr/usagers/politique-dutilisation/ 


\section{«La poésie dans la prose, ou le clochard illuminé»}

\section{par André Brochu, Université de Montréal}

Le lecteur qui aborde pour la première fois l'œuvre en prose de Jacques Brault risque d'être surpris par l'atmosphère d'apparente morbidité qui s'en dégage. Et si je m'exprime ainsi, ce n'est pas pour suggérer que les apparences cacheraient leur contraire. qu'une jovialité truculente fleurirait sous les cercueils. 11 y a indubitablement une fascination pour la mort chez Jacques Brault. Seulement, on ne peut la réduire à une particularité psychologique, encore moins l'assimiler à quelque trait pathologique. Elle est un système complet d'aperception du monde et contient de ce fait beaucoup plus qu'ellemême, elle rejoint toutes les dimensions de l'existence.

L'œuvre narrative de Jacques Brault comprend trois Nouvelles' publiées au début des années soixante (avant "Suite fraternelle» et "Mémoire ${ }^{2}{ }^{2}$, qui ont révélé le poète), Chronique de l'autre vie (extrait) ${ }^{3}$, parue en 1972, et surtout Agonie ${ }^{4}$ qui a valu à son auteur, en 1984, le Prix du Gouverneur général. On pourrait y joindre les textes dramatiques regroupés sous le titre de Trois partitions ${ }^{5}$ (1972); d'ailleurs la première des nouvelles de 1963 . «L'envers du décor», recourait à la forme dramatique (dialogue, accompagné d'indications de régie).

Poésie, dialogue, récit sont trois formes d'écriture intimement liées chez Jacques Brault, qui ne peut guère concevoir une prose - qu'elle soit narrative ou dramatique - sans l'inclusion d'un ou de plusieurs textes poétiques. «L'envers du décor» (Nouvelles) met dans la bouche d'Albert, le personnage principal, un monologue (récité recto tono) formé de vers rimés, puis une chanson de style médiéval, exactement versifiée. La nouvelle suivante, "Le narrateur", insère dans le récit des poèmes en vers libres qui sont comme des commentaires lyriques sur certains personnages. La dernière des nouvelles de 1963. "Celle qui sera", la plus longue et la plus riche. fait exception à la règle par l'homogénéité de son écriture. Elle contient cependant des passages d'un lyrisme appuyé, comme le suivant: Toi l'entrevue, la présente furtive. toi la tournolante, la promise fugace. l'enfuie et la revenue, ah! que tout se fige, que les choses prennent, et toi et moi dedans, nous appelant, nous possédant à distance (N, p. 93).

Chronique de l'autre vie fait référence à beaucoup d'auvres du passé, entre autres à la Chanson de Roland, et donne une grande valeur stratégique à deux citations du "Livre" (la Bible?), aux saveurs amères, qui rappellent l'Ecclésiaste. 
Les Trois partitions intègrent la poésie de façon encore plus poussée. Dans "La morte-saison", court téléthéâtre, l'auteur imagine un Roméo et une Juliette bien de chez nous, trente ans après leur mariage. La référence au grand chef-d'cuvre de Shakespeare est partout présente et devient explicite vers la fin, quand les deux personnages récitent la scène de Juliette au balcon. Le désir de recouvrer leur amour et leur jeunesse détermine leur suicide. La poésie a donc une action décisive sur le destin des personnages. "Quand nous serons heureux", téléthéâtre de plus grande envergure, met en scène une sorte d'innocent jovial. Félix, seul capable d'entendre, sur son transistor brisé, une chanson qui est un véritable poème et dont les modulations thématiques ponctuent chaque moment de l'action. La "Lettre au directeur ", radiothéâtre, présente un employé de bureau en fin de carrière qui voudrait devancer la retraite en remettant sa démission. Il en est empêché par les objurgations inattendues de la femme de ménage qui ne veut pas être privée des beaux moments où il récite des poèmes, pendant qu'elle travaille en silence. La poésie intervient donc au sein même de l'activité la plus quotidienne, pour en orienter les dispositions.

Mais c'est Agonie, l'objet principal de la présente étude, qui réussit l'intégration la plus complète du poème et du récit. Tout le récit, en effet, se présente comme une expansion narrative d'un poème d'Ungaretti cité en exergue, et dont chaque vers sert de titre aux chapitres du livre. Le déroulement du poème correspond donc exactement à celui du discours náratif. Cette particularité formelle nous servira de point de départ pour notre interrogation sur le sens.

La structure textuelle - ou, si l'ón préfère, la texture - d'Agonie est fort complexe puisque le récit, avec ses dimensions particulières (diégèse, discours narratif, narration ou acte de raconter) est en ràpport avec un autre texte, qui appartient au genre poétique et qui comporte lui aussi ses dimensions propres. Celle du sens cependant est privilégiée, et exposée dans un commentaire qui fait partie du récit. (Le commentaire du poème est tantôt le fait du personnage principal et relève alors de la diégèse; tantôt le fait du narratéur et procède de l'acte narratif).

Puisque chaque vers du poème sert de titre aux divisions du récit. on peut affirmer que l'ordre des segments du discours poétique correspond à celui des segments du discours narratif, que les deux syntagmatiques textuelles coïncident rigoureusement - quitte à préciser la nature de l'adéquation pour chaque couple de segments. Mais la succession des segments du discours narratif ne correspond pas nécessairement à celle des événements qui composent la diégèse, c'est-à-dire l'histoire racontée. Du reste, il y a deux histoires: celle du personnage principal. ce professeur de philosophie qui devient clochard, et celle du narrateur, son élève, qui enquête sur le destin de l'autre. L'intervention de l'énonciateur donne consistance à la narration proprement dite, qui prend place (en position terminale, bien entendu) parmi les 
événements de la diégèse. Ce dispositif n'est pas sans rappeler fortement l'écriture autobiographique, notamment celle du journal intime, où les actes de narration s'insèrent entre les événements racontés et sont souvent euxmêmes thématisés. La narration, dans Agonie, est en position terminale dans la diégèse mais elle n'en est pas moins segmentée, chaque chapitre correspondant à un moment distinct. Entre ces moments. on suppose logiquement l'activité du narrateur prenant connaissance des renseignements dont il peut disposer sur la vie du personnage-objet.

Autre facteur de complexité. Les vers du poème ont une charge symbolique qui les rend aptes à illustrer divers événements de la vie du personnage principal; mais ils sont aussi, de sa part, l'objet d'un commentaire qui se situe dans un segment déterminé de la diégèse, soit les derniers cours de philosophie qu'il a faits (et auxquels le narrateur a assisté). Tout se passe alor's comme si le professeur avait commenté à l'avance des événements de sa vie à venir.

Comme si les relations complexes engendrées par les deux dispositifs textuels, poème et récit, ne suffisaient pas, il faut tenir compte d'un autre texte, dont le professeur est l'auteur et où le narrateur puise l'essentiel de ses renseignements biographiques: le journal, sous forme de carnet gris, qui contient des notes éparses et qui a suivi le personnage principal jusque dans sa vie d'itinérant, après avoir été matière à rigolade pour ses élèves peu indulgents.

Comment s'articulent tous ces éléments? La lecture, pas à pas, de ce "roman" dense mais bref permettra de s'en faire quelque idée.

D'abord figure en exergue le poème d'Ungaretti, dans la traduction de Jean Lescure. Il porte le même titre que le roman. Ses trois courtes strophes évoquent chacune un oiseau: les deux premiers, l'alouette et la caille, savent mourir, au bout de l'effort fourni ; le troisième, le chardonneret aveugle, se contente de vivre de plaintes. En somme, il vaut mieux mourir d'avoir pleinement vécu, que de vivre à moitié. On pourrait aussi comprendre: rien ne sert, une fois atteint par la vieillesse ou la maladie. de prolonger une vie qui n'en est plus une, d'éterniser l'agonie. L'agonie doit faire nettement et succintement le partage entre la vie et la mort, elle doit être un acte. non un état où l'on s'installe à demeure. Le refus des plaintes rejoint la morale stoïcienne - qui s'exprimait déjà. chez un Vigny, à travers une symbolique animale ( La mort du loup»).

Le récit commence à ce point de l'histoire où le narrateur, inscrit à des cours de philosophie, entend plus ou moins son professeur prononcer une parole inquiétante, qui le réveille net. Cette parole. cependant, il lui faudra tout l'espace du récit pour s'en souvenir puisqu'elle ne sera révélée qu'à la dernière page (p. 77). Elle joue donc le rôle d'une énigme, et constitue sans doute un élément important du texte, porteur d'un signifié qui, d'être ainsi différé,'se présente comme une clé. Cette phrase, commentons-la dès maintenant, est la belle et pure expression du défaitisme national, ce défaitisme qui 
est sans doute le moteur réel de ce magnifique rêve d'indépendance auquel les écrivains québécois ont voulu croire pendant quelques années, pour mieux s'enchanter ensuite de leur impuissance et de leur défaite. C'est: $/ / n^{\prime} l^{\prime}$ a pas, il n'y a jamais eu, il n'y aura jamais de pay's. Rien, en un sens, ne prépare cet énoncé puisque le récit ne fait aucune part à l'idéologie souverainiste ou nationaliste, il remue plutôt les grands thèmes universels que sont l'amour et la mort, d'ailleurs inscrits dès les poèmes de Trinôme' dans l'œuvre de Jacques Brault. Et pourtant, on n'est pas surpris que l'auteur de Mémoire se souvienne du rêve québécois, ne serait-ce que pour le donner à désavouer à son personnage: l'échec du moi n'étant pas seulement celui de tout homme. mais aussi et plus précisément celui de tout Québécois. L' "agonie» symbolise ainsi- - virtuellement - la mort du pays c'est-à-dire l'assimilation du peuple québécois, lequel doit cesser de vivre de plaintes et se faire une raison: disparaître. Pour un peuple comme pour tout individu, il y a plus de dignité à mourir qu'à vivre diminué. Et, bien sûr, l’auteur n’énonce ici aucun précepte, aucun enseignement. Le sens que j'indique est un sens possible, inscrit dans une logique des choses que l'écrivain se contente de faire apparaître, sans aucunement l'imposer. Ce sens n'est sûrement pas le seul possible même si, aujourd'hui, il peut sembler plus plausible qu'aucun autre. Si Agonie est un chant de défaite, s'il connote l'échec d'une collectivité qui, par bêtise ou impuissance, a choisi le suicide, c'est aussi parce que la défaite est une victoire sur l'insignifiance, que le suicide est préférable à l'infâme compromis d'une existence châtrée.

Mais trêve d'anticipation. Les premières lignes m'ont projeté vers les dernières - le début est déjà la fin. Entre l'un et l'autre, il y a cependant tout l'espace du récit. de l'œuvre, de la vie - et rien n'y évoque spécifiquement, je le répète, la réalité nationale.

L'action commence donc par la profération d'une parole, en fin de cours, parole qui échappe à la perception claire du narrateur mais non à sa perception seconde puisqu'elle déclenche en lui le dégoût et détermine même le vomissement. Le récit de cette action s'accompagne d'une présentation du professeur, gris et malingre, l'air absent, timide, idiot, minable: un anti-héros s'il en est un, jamais nommé. et auquel il n'est pas question de s'identifier. Le narrateur, anonyme lui aussi, pourtant le fera mais graduellement. et à son corps défendant.

Il est ensuite question du carnet gris que le narrateur vient, dix ans plus tard, de subtiliser. Le récit présente donc, côte à côte, deux moments éloignés de la diégèse, le deuxième étant tout proche, semble-t-il, du moment de la narration. Après quoi on revient au passé: le narrateur s'inscrit au cours de scolastique du professeur. Le baccalauréat spécialisé lui permettra, espère-t-il, de devenir un grand reporter (en fait, au moment où il écrit, il végète depuis cinq ans dans une petite agence de publicité, son destin est aussi minable que celui de l' "homme gris"). Le cours portera sur les universaux.

Autre prolepse. qui vient simplement préciser la précédente, l'enrichir de détails nouveaux: nous sommes en octobre, le narrateur vient de rencontrer 
son ancien professeur qui était seul sur un banc du parc Lafontaine. et de prendre son carnet gris posé à côté de lui. La narration a lieu pendant la même nuit. dans l'après-coup du vol du carnet qui déclenche l'enquête sur son propriétaire.

Retour au passé. Le professeur va consacrer les dernières séances du cours au beau - et aussi à la beauté. Pour expliquer la différence entre les deux. il lit. dans son carnet. le poème d'Ungaretti, dont il commentera un vers'à chacun de ses cours. Et il commente le premier: Mourir comme des mouettes altérées. Le narrateur reproduit "in extenso" le commentaire. sans le mettre en relation avec la vie de son auteur. comme ce sera le cas dans les chapitres subséquents. On ne sort de la glose que pour en parcourir le théâtre: ce professeur au visage ravagé comme celui d'un enfant, un vieil enfant obscène. la fenêtre où grésille une feuille sèche rabattue par le vent.

L'essentiel du commentaire porte sur le fait de commencer le poème par le mot mourir, qui signifie la fin. Mourir, acte initial plutôt que terminal. $\grave{A}$ rapprocher de l'expression vieil enfant obscène, qui suggère symétriquement la subsistance du début dans ce qui va fínir. Si la vie est d'emblée la mort. toute l'existence n'est qu'une agonie prolongée. laquelle maintient l'origine qu'elle nie. et pour mieux la nier. L'agonie est alors synonyme de cette survivance dont le "Canada français" - c'est moi qui commente - a fait son devoir (glorieux, abject). Le vieil enfant obscène. c'est cet homme Plutôt jeune et plutôt vieux qui. dans un poème de Saint-Denys Garneau. "Commencement perpétuel", réinvente le mythe de Sisyphe en recommençant continuellement. sans succès, à compter jusqu'à cent. Ce temps de l'éternel retour, de l'échec inlassablement répété. c'est celui même de la survivance; et comme il ne peut déboucher sur la vie. la mort seule, qui l'inaugurait. pourra $y$ mettre un terme.

A propos d'"échec" - que traduit le mot flop en québécois - je note que la récitation du poème par le professeur le réalise phonétiquement puisque chaque mot se détachait sur un fond de silence lourd. Cela faisait flop-flop. comme des pas qui s'arrachent à la vase. Au début de tout. chez Jacques Brault il y a cet enlisement. cet engluement dans une matière indécise entre le solide et le liquide. état crépusculaire où tout est à déméler. vie et mort, amour et mort. Le flop-flop du discours professoral plonge le narrateur (alors son étudiant) dans un véritable état d'hypnose. où point sans doute. mêlée au dégoût. l'espèce d'affection qui le liera au "petit homme gris".

Le deuxième chapitre raconte d'abord les circonstances qui ont permis au narrateur de revoir son ancien professeur. La présentation dun film sur le Népal, à la salle du Plateau, a attiré celui qui rêvait de devenir reporter. Dans l'assistance, il reconnait soudain son ancien professeur de heaulé. le minable pour qui jéprouvais attirance et répulsion. II se souvient alors du commentaire sur le deuxième vers: "Sur le mirage", dont la sémantique est en harmonie avec lagitation colorée de Katmandou. Le commentaire ressouvenu. d'abord lexical et proche du texte. dérive bientôt vers la situation présente. et l'alouette altérée qui meurt sur le mirage symbolise maintenant la relation $\mathrm{du}$ 
narrateur au héros (professeur): Et je vois cet homme navré qui m'étonne. Je suis une alouette devenue étrangère à elle-même et qui se mire dans un my'stère. Ce mystère, c'est sans doute l'autre pour autant qu'il est la mort, le pays de l'ailleurs (Népal), le non-pays.

Percevant l'autre comme un mystère, le narrateur imagine que ce dernier a subi une illumination semblable à celle du tantrisme et qu'elle est le secret de sa vie, secret qu'il lui faut chercher par delà les apparences mensongères. De là le projet d'une enquête et le vol du carnet gris.

Après la représentation. le narrateur suit l'homme-mirage, n'ose l'aborder. prend son carnet (le récit nous ramène au même événement pour la troisième fois, sans que le caractère répétitif du récit soit thématisé, ce qui peut créer quelque confusion dans l'esprit du lecteur) et rentre chez lui.

Le poème, on le voit, n'est plus seulement inscrit dans un moment éloigné de la diégèse, il colore maintenant le passé récent, définit la vérité de la relation actuelle entre le narrateur et le héros.

Le vers suivant, $\mathrm{Ou}$ comme la caille, génère un autre segment narratif qui commence par une réflexion du narrateur sur le carnet, qu'il est tenté de rendre à son auteur (ce qui mettrait fin à la narration), et se poursuit par l'évocation, fondée sur des renseignements passablement lacunaires, du passé du professeur: ses études, sa carrière universitaire, son existence de célibataire vivant avec sa mère, vie minable (comment mourir quand on ne vit pas?) et semblable à celle du narrateur lui-même, où les rares échappées de tendresse se soldent par un retour au délabrement. Le narrateur et son héros se rejoignent dans une même "agonie». La connaissance de l'autre, en particulier dans ses aspects de tendresse et de fragilité (que symbolise la caille), provoque une vertigineuse identification.

C'est par une très libre association d'idées qu'est introduit cette fois le commentaire du poème. Le narrateur s'imagine présent à un pique-nique du professeur avec sa mère, présent et dissimulé comme la caille du troisième vers. La caille, selon le commentaire du professeur, est assimilable à la femme, à l'enfant, aux êtres fragiles que menace le désir meurtrier. La fin du chapitre fait allusion, entre autres choses, à la disparition du professeur, à la fin du cours: il a reçu une incrolable invitation en Europe. Beaucoup de propos aussi sur le marasme du narrateur lui-même, qui n'a rien à envier à celui de son héros (c'est faute de nom propre que je me vois réduit à utiliser cette expression, fatalement dérisoire). Il désespère de connaître jamais une illumination soudaine au coin de la rue quand je reviens ecouré du bureau. Voilà un schème fréquent chez Jacques Brault, celui du contre-enlisement qui se réalise dans la mort fulgurante, la mort qui est toute vie et qui est connaissance totale, douceur infinie, amour. Cet instant transformateur se loge nécessairement dans la vie la plus plate. la plus répétitive, la plus agonique, et elle justifie donc le choix du plus grand conformisme - vie conjugale, par exemple: J'aurais dû me marier, avoir des enfants. Je travaillerais dur et petitement, pour quelqu'un (p. 30). Cette vie, c'est la prose la plus étroite, au cœur de laquelle éclate soudain le miracle poétique. 
Le chapitre intitulé "Passée la mer", grâce aux révélations du carnet, commence à combler l'intervalle de dix ans qui sépare les derniers cours du professeur de la nuit présente, où le narrateur l'a revu. En plein mai 1968. notre héros se rend à un congrès de latinistes au lac d'Annecy. Un rapport métaphorique s'établit tout naturellement avec le quatrième vers. et le professeur (ou est-ce le narrateur?) commente l'héroïsme inutile de l'oiseau dont l'effort aboutit à la mort - image, probablement, de toute existence.

Suit le récit du départ par le train de nuit; puis l'évocation du temps de la narration, où le narrateur réfléchit sur la solitude du professeur et sur la sienne propre: le destin de l'autre est de plus en plus mêlé au sien, et la logique de cette gémellation, elle est dans le poème qui fonctionne comme un universel, intégralement applicable à l'un et à l'autre, à tout être vivant. Telle est la vérité du poème: il dit l'essentiel de chacun - comme si, en chacun. était l'essentiel; et comme si l'essentiel faisait le particulier, non l'inverse.

(Ainsi, ce n'est pas d'être tel pays qui fait du Québec ce qu'il est, mais d'être en instance de mort, de vie, d'amour.)

L'absence de nom du héros, du narrateur, est peut-être la marque de leur universalité, tout en étant celle de leur inexistence. de leur vie agonique. La mort serait alors la recouvrance de soi (p. 34). donc le lieu où l'on peut advenir à son nom.

Les tribulations de la traversée, de l'arrivée à Southampton puis dans Paris soulevé, enfin de la fuite vers la Hollande occupent le reste du chapitre et, pour la première fois, une stabilité narrative s'installe (il est vrai, dans le récit de l'instable). Pendant trois pages au moins. le discours narratif suit l'ordre chronologique des événements.

Cet ordre est rompu et, grâce au cinquième vers («Dans les premiers buissons"), on est ramené vers l'enfance du héros qui, avant l'âge de neuf ans. était déjà tout gris en dedans (p. 37). Au sein de la détresse quotidienne. continue, un seul rayon de soleil: Michèle, la petite voisine. Bonheur buissonnier. Mais elle déménage et il ne reste rien. Rien que l'absence d'enfance. Dans son commentaire, le professeur fait du buisson le coin d'enfance retrouvée où la bête se cache pour mourir. C'est dire, une fois de plus, que la mort est un acte et un bonheur, un pied de nez à la grisaille des jours. Autre épisode, qui prend place dans l'adolescence. Au cours d'une excursion avec des camarades du collège, dans un restaurant, il rencontre une jeune fille blonde qui est la réincarnation de Michèle. Elle est la promesse retrouvée (p. 42). Pas question d'établir avec elle de vrajes relations: elle habitera ses rêves, sans plus. À vingt ans, il se glisse dans une défroque, entre au séminaire. C'est l'époque de la deuxième guerre mondiale.

Parce qu'elle n'a plus désir: le sixième vers dit la mort du désir, qui prélude à la mort tout court. Dans la vie du professeur, il correspond au retour en Amérique, après l'épisode de Rotterdam qui sera raconté au chapitre suivant. Il correspond aussi à la mort de la mère, que le professeur, parti pour la France, avait laissée gravement malade derrière lui. La mort de la mère. 
jointe à une aventure en Hollande dont on connaîtra plus loin la nature. détermine cette capitulation qui transforme en "semi-clochard" notre professeur de philosophie. Plus de désir, donc plus de rôle social, de façade. d'extérieur. Chez Jacques Brault, être clochard (et c'est le destin de ses personnages les plus raffinés, depuis ses tout premiers récits!), c'est vivre selon sa seule exigence intérieure, totalement libre mais en même temps imbibé complètement de la grisaille des choses, devenu docile à l'extrême prose de l'existence.

De voler: le vol de la caille, de la bécasse, c'est la vie, l'amour. C'est, dans la vie du professeur, l'instant de grâce de la rencontre amoureuse avec cette femme de Rotterdam qui réveille le souvenir de Michèle et de sa réincarnation en serveuse blonde; qui invente un roman pathétique pour cacher son identité de prostituée. Le récit lui-même prend son envol, atteint une grâce et une aisance que ne viennent pas briser, pour une fois, les évocations du réel. On atteint sans doute ici un sommet dans la vie du personnage et, en tout cas, dans sa vie amoureuse puisque la "promesse" est enfin réalisée, pour la première et la dernière fois. Est-ce bien le moment d'illumination que recherchait le narrateur? Il faut dire que ce chapitre, le plus long du roman, avait commencé sur une note particulièrement sombre: l'échec du personnage principal, vécu plus intensément que jamais à Bruxelles, et l'engourdissement frileux du narrateur, qui se désole à la pensée de l'autre abandonné sur son banc, par une nuit glaciale. Par contraste, les brèves amours hollandaises acquièrent beaucoup de relief. Comme le vol de la caille, qui les figure poétiquement, elles sont coextensives à toute une existence en ce que, au-delà, il n'y a plus que la mort. Ou une indigne survie.

C'est cette survie qu'évoque l'avant-dernier chapitre, "Mais non pas vivre de plaintes», où plus que jamais le narrateur se projette sur l'hommeécran qui le hante, homme qui ne (lui) est rien mais qui ressuscite peut-être pour lui son père bafoué - Chronique de l'autre vie évoquait aussi le souvenir d'un père chômeur, qui se laissait battre par le voisin devant son fils en pleurs. Survivre à une telle honte, c'est s'installer dans la plainte (comme le Québec dans son interminable défaite), dans la contagion de la douleur. Le narrateur est touché (ll aura fallu que ce petit homme gris trottine comme une souris dans quelque recoin de ma vie, et me voilà face au néant, un néant falot, un néant de doux raté. Je me plains...), comme l'a été avant lui rinfirmier qui a recueilli le professeur tombé dans la complète clochardise et terrassé par une attaque.

Mais lui, l'homme gris, tout en survivant - au voyage, à l'amour, mais aussi à la mort de sa mère - . s'installe dans un au-delà de la plainte qui est peut-être la mort. qui est la mort sociale en tout cas, et qui ressemble à un bonheur. La clochardise est un sacerdoce et une transfiguration: tout en se dépenaillant, en subissant une dégradation physique, l'homme est comme éclairé de l'intérieur, son visage ravonnait sans raison apparente, il s'illuminait (p. 69). Le clochard illuminé, c'est l'en dessous devenu l'admirable, le soushomme qui triomphe ailleurs, qui a laissé derrière lui cette humanité dont le lot quotidien est un pâteux malaise, une boue où l'on farfouille péniblement. Et voilà sans doute le secret que cherchait le narrateur: le "grand refus" de 
tout, le silence. Par lui, les traces de la vie sont effacées: une sublimation si légère que là où il n'était plus il n'avait pas été. Tel était l'enseignement de ce Zarathoustra cloporte. Le clochard est le seul véritable mystique occidental.

Un dernier chapitre vient faire le raccord entre le passé et le présent. Sorti de l'hôpital où il s'est remis de son attaque, le professeur-clochard décide d'assister à un film sur le Népal... ce qui nous amène au point précis où commençait le récit, et au début de cette nuit où s’élaborera la narration elle-même. Le commentaire sur le "chardonneret aveugle» se rapporte à la problématique du retrait intégral exposée dans le chapitre précédent. Tout est bouclé, diégèse. récit et narration, et pourtant, le titre du poème, "Agonie". n’a pas encore été commenté. C'est alors que la phrase: Il n'y a pas, il n'y a jamais eu, il n'y aura jamais de pays est énoncée, et elle commente à elle seule ce mot d'uagonie" dont tout le poème, en fait, et par delà le poème, tout le récit sont les commentaires par le vécu.

Si l'agonie est la décision de mourir, d'outrepasser l'existence plaintive, elle fait du clochard un être lumineux (il titube dans sa nuit comme la flamme d'une chandelle). Du même coup, une raison supérieure est introduite dans la vie de tous les jours, et c'est celle même du poème, illuminant la prose du récit. Tout ce roman, très court et très long, serait la lamentable description de la vie lamentable sans cette référence à une langue réservée, la poésie, qui tire la beauté de cela même qui la nie, qui change les infinies disgrâces du réel en images nécessaires. Et même les destins de l'homme, de l'individu, d'un peuple, d'un pays, si navrants soient-ils, deviennent matière à cette réflexion transfigurante qu'est la prose du poète, la prose ébranlée de poésie, ouverte à une angoisse d'être, soudain devenue merveilleuse.

1. André Major, Jacques Brault et André Brochu, Nouvelles, Montréal. Cahiers de l'A.G.E.U.M., no 6, 1963, 140 p. Les nouvelles de J. Brault sont aux pages 69 à 104.

2. Ces deux poèmes ont été réunis dans Mémoire, Montréal, Déom. 1965. $80 \mathrm{p}$.

3. Liberté, no 81, Montréal, juillet 1972, p. 88-102.

4. Publié d’abord aux éditions du Sentier, Montréal, 1984. Repris par Boréal Express, Montréal, 1985, $78 \mathrm{p}$.

5. Montréal, Leméac, $1972,196 \mathrm{p}$.

6. Richard Pérusse, Jacques Brault et Claude Mathieu. Trinôme. Montréal, Jean Molinet, 1957, 58 p. Les poèmes de J. Brault, "D'amour et de mort", sont aux pages 21 à 39 . 


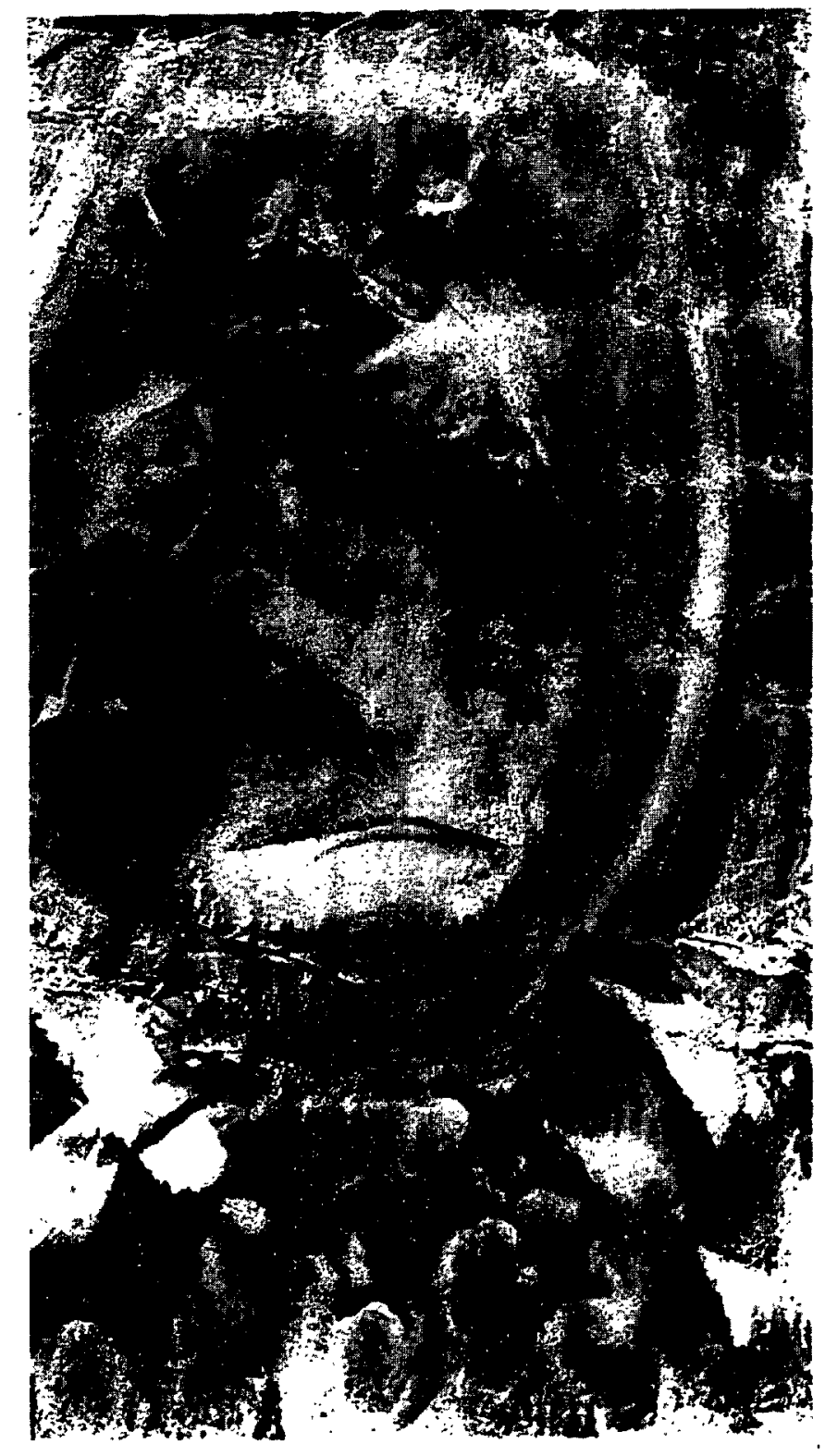

...une angoisse d'être, soudain devenue merveilleuse. 